\title{
Manifestaciones pulmonares y radiológicas del Sars-Cov2 en pediatría.
}

\author{
Pulmonary manifestations and radiological findings of SarsCov2 in children
}

*Wilmer A. Madrid, ** Gabriel Madrid-Mejía, ** Miriam

Sánchez Luna, ${ }^{* * *}$ Laura Gochicoa-Rangel.

\section{RESUMEN}

Las infecciones respiratorias agudas son causa de alta morbi-mortalidad a nivel mundial. El Coronavirus descubierto en Wuhan, China en el 2019 es de interés mundial por las diferentes manifestaciones clínicas que pueden aparecer en niños. Nuestro objetivo principal es revisar las manifestaciones del COVID-19 en el aparato respiratorio y los hallazgos a nivel radiológicos en pediatría. Se estiman que solo el 5,7\% de los niños han sido hospitalizados, siendo los niños con comorbilidades y menores de 1 año los que suponen un riesgo mayor para enfermedad grave. A nivel global la mediana de edad de presentación de los casos es alrededor de 7 años, detectándose más casos a mayor edad, pero siendo la gravedad inversamente proporcional a la edad. La forma asintomática o leve son las presentaciones más comunes reportadas en niños, siendo de hasta en el $90 \%$ de los niños infectados. Los estudios de laboratorio pueden ser normales o presentar leucocitos alterados en un $69,2 \%$ y a nivel radiológico se ha observado en niños que en un $69 \%$ presentan opacidades bilaterales en vidrio despulido. En este momento, cualquier infección

1. Neumólogo Pediatra Instituto Nacional de Pediatría, Alta especialidad en fisiología respiratoria Instituto Nacional de Enfermedades Respiratorias

2. Estudiante de Medicina Universidad Católica de Honduras

3. Médico General graduado de la Universidad Católica de Honduras

4. Neumóloga Pediatra, Jefe de departamento de Fisiología respiratoria y laboratorio de función pulmonar Instituto Nacional de Enfermedades Respiratorias, Instituto de Desarrollo e Innovación en Fisiología Respiratoria S de RL (INFIRE). Correspondencia: wmadridneumologo@gmail. com Recibido: 20 de Mayo de 2020 Aprobado: 20 de Septiembre de 2020 respiratoria aguda hay que descartar COVID-19, para poder evitar complicaciones en el paciente.

\section{PALABRAS CLAVE}

Coronavirus, pneumonia, 2019-nCoV, children, COVID-19, SARS-CoV2

\section{ABSTRACT}

Acute respiratory infections are a cause of high morbidity and mortality worldwide. The novel Coronavirus discovered in Wuhan, China in 2019 is of worldwide interest due to the different clinical manifestations that can appear in children. Our main objective is to review the manifestations of COVID-19 in the respiratory system and the radiological findings in pediatrics. It is estimated that only $5.7 \%$ of children have been hospitalized, with children with comorbidities and under 1 year of age being at greater risk of severe disease. Globally, the median age of presentation of cases is around 7 years, with more cases being detected at older ages, but with severity inversely proportional to age. The asymptomatic or mild form of disease are the most common presentations reported in children, being up to $90 \%$ of infected children. Laboratory studies can be normal or present abnormal leucocytes in $69.2 \%$ of the cases. At a radiological level, it has been observed that $69 \%$ of the children present with bilateral ground-glass opacities. At this time, in any acute respiratory infection COVID-19 must be excluded in order to avoid future complications in the patient. KEYWORDS

Coronavirus, pneumonia, 2019-nCoV, children, COVID-19, SARS-CoV2 


\section{INTRODUCCIÓN}

Las infecciones respiratorias agudas son causa de alta morbi-mortalidad a nivel mundial especialmente en menores de 5 años, esto nos lleva a conocer nuevos virus emergentes en el mundo como es la enfermedad por el coronavirus descubierto en el 2019 (COVID-19) en China. En esta revisión nuestro objetivo principal es revisar las manifestaciones del COVID-19 en el aparato respiratorio y los hallazgos a nivel radiológicos en pediatría. El COVID-19 es un virus de ARN monocatenario envolvente, de la familia coronaviridae del genero beta. Su nomenclatura surge de las proteínas; superficial (S) que dan la apariencia de "corona" (1,2) y se le conoce como virus del síndrome respiratorio agudo grave causado por coronavirus (SARS-CoV-2). El sistema respiratorio es la principal puerta de entrada para la infección por SARS-CoV-2 a través de la proteína $S$ que se une a los receptores angiotensina 2 (ACE2), dicho receptor se encuentra en la membrana basal del epitelio de la mucosa nasal, oral, nasofaríngea, orofaríngea, epitelio alveolar, vasos sanguíneos, corazón, túbulos renales y enterocitos ${ }^{(3)}$. Una vez que se une al receptor ingresa a la célula donde se produce la primera etapa de replicación viral (periodo de incubación de 1-14 días), posteriormente aparece la etapa de respuesta inmune que se caracteriza por afección pulmonar con aparición de infiltrados pulmonares y elevación de marcadores de inflamación y finalmente ocurre la etapa donde podemos encontrar falla multiorgánica. ${ }^{(4)}$ Datos del Centro de Control y Prevención de enfermedades (CDC) en Estados Unidos de América (EUA) estiman que solo el 5,7\% de los niños han sido hospitalizados, siendo los niños con comorbilidades y menores de 1 año los que suponen un riesgo mayor para enfermedad grave. Entre países existen diferencias estadísticas, por ejemplo en España se han hospitalizado alrededor del $60 \%$ de los niños por síntomas leves y el $9.7 \%$ ha requerido terapia intensiva, mientras que en China entre los niños sintomáticos, el $5 \%$ tenía disnea o hipoxemia y menos del $1 \%$ progresaba a enfermedad grave (5,6). A nivel global la mediana de edad de presentación de los casos es alrededor de 7 años, detectándose más casos a mayor edad, pero siendo la gravedad inversamente proporcional a la edad. En una revisión de pacientes la proporción de casos graves fue de $10,6 \%, 7,3 \%, 4,2 \%, 4,1 \%$ y $3,0 \%$ para los grupos de edad, 1, 1-5, 6 - 10, 11-15 y mayores 15 años, respectivamente, lo que indica que a menor edad mayor gravedad. ${ }^{(7,8)}$ Los síntomas a nivel respiratorio y gastrointestinal son los de presentación mas frecuentes, Tu-Hsuan Chang et al, mediante un meta-análisis describió que más el 59\% de los pacientes presentaron fiebre, $46 \%$ tos y sólo el 12\%, manifestaciones gastrointestinales. Aproximadamente el $26 \%$ de los pacientes no mostraron inicialmente ningún síntoma específico. Los pacientes pueden manifestar otros signos y síntomas respiratorios que afectan en menor frecuencia como eritema faríngeo, rinorrea, congestión nasal y desaturación. ${ }^{(7,8)}$

Manifestaciones clínicas:

- Infección asintomática - estos niños presentan una prueba PCR positiva a SARS-CoV-2, pero sin síntomas clínicos y radiológicamente sin ninguna anormalidad en tórax.

- Infección aguda del tracto respiratorio superior - se manifiestan con tos, dolor faríngeo, fiebre, obstrucción nasal, fatiga, dolor de cabeza, mialgia o malestar general. No hay evidencia de neumonía ni clínica ni radiológicamente.

- Neumonía leve - puede haber o no fiebre $y$ se acompaña de síntomas respiratorios, las imágenes radiológicas muestran datos de neumonía no grave.

- Neumonía grave - el paciente presenta alguna de las siguientes manifestaciones: 1) aumento de la frecuencia respiratoria ( $\geq 70$ latidos $/ \mathrm{min}$ en menores de 1 año, $\geq 50 \mathrm{~L} / \mathrm{min}$ mayores de 1 año); 2) saturación de oxígeno <92\%; 3), alguna manifestación de hipoxia severa como alteración del estado de conciencia, rechazo a 
- la alimentos o dificultad de alimentación y; 4) signos de deshidratación. Puede presentarse en un $2.5 \%$ de los pacientes infectados

- Niños en estado critico - son aquellos niños que requieren monitoreo de UCI por cumplir presentar manifestaciones de insuficiencia respiratoria que requirió ventilación mecánica, choque o falla multiorgánica. La mayoría de niños que se encuentran en esta etapa presenta alguna comorbilidad, y puede presentarse en el $0.4 \%$ del total de pacientes. ${ }^{(7,9,10)}$. La forma asintomática o leve son las presentaciones más comunes reportadas en niños, se ha reportado que hasta en el $90 \%$ de los niños infectados se manifiestan de esta manera, el $2.5 \%$ presentarán neumonía grave, y alrededor de un $0.4 \%$, ingresarán a la unidad de cuidados intensivos. ${ }^{(1)}$ Existen diferencias en la presentación de los síntomas entre niños y adultos, esta condición ha sido explicada mediante diversas teorías. Como ha podido observarse los niños cursan asintomáticos o con síntomas leves y la mortalidad es menor, una de las causas que pueden explicar este fenómeno es la baja expresión de receptores de ACE2 en comparación con los adultos. ${ }^{(12)}$ El estímulo inmunológico también ha sido planteado como un mecanismo protector, ya que los niños presentan inmunidad obtenida por una estimulación antigénica ya sea por infecciones o vacunación previa, se ha postulado, por ejemplo, que la vacuna BCG, puede generar que las células inmunitarias innatas pasen a un estado más activado por la estimulación ${ }^{(12)}$. La inmunidad cruzada podría tener algún papel protector de los anticuerpos de otras infecciones por coronavirus contra el SARS-CoV-2. La respuesta adaptativa inadecuada, en niños de menor de edad podría explicar el peor curso de la enfermedad por COVID-19 en comparación con los niños mayores y con los adultos. Los niños tienen buena capacidad regenerativa y mejor recuperación pulmonar al presentar COVID-19, la presencia de una mayor resistencia en las vías respiratorias superiores en los niños podría causar que las partículas de aerosol se depositen más en el árbol traqueobronquial que en los alvéolos, y otro factor muy importante son las comorbilidades de los adultos, ya que presentan diversas patologías que podrían explicar la susceptibilidad a presentar más complicaciones secundarias SARS-CoV-2 ${ }^{(12)}$

\section{-Hallazgos laboratoriales y patológicos:}

A nivel patologico, Luca Carsana et al en Italia, describieron que los pacientes fallecidos por SARS-CoV-2 presentaban a nivel pulmonar, daño alveolar difuso, congestión capilar, edema intersticial, necrosis de neumocitos, membrana hialina, hiperplasia de neumocitos y atipia reactiva, trombos de plaquetas-fibrina y se observó que también tenían un infiltrado inflamatorio compuesto por macrófagos y linfocitos. ${ }^{(13)}$ En lo laboratorial, el SARS-CoV-2 se ha demostrando la existencia en el organismo ya sea por la reacción de cadena de polimerasa (PCR), detección de antígeno del virus o por la inmunidad que produjo al ser expuesto. Wang y col, describieron que la sensibilidad de detección para SARS-CoV-2 mediante PCR en muestras provenientes de diferentes sitios del sistema respiratorio en adultos era el siguiente: lavado bronquioalveolar $(14 / 15 ; 93 \%)$, esputo $(75 / 104$; $72 \%)$, hisopado nasal $(5 / 8 ; 63 \%)$, biopsia por cepillado bronquial $(6 / 13 ; 46 \%)$, hisopado faríngeo $(126 / 398 ; 32 \%)$, heces $(44 / 153 ; 29 \%)$, sangre $(3 / 307 ; 1 \%)$ y orina $(0 / 72)$ REF. En un niño sintomático, se espera que la carga viral sea elevada y una sola prueba con una muestra de las vías respiratorias superiores (hisopo nasofaríngeo y orofaríngeo) debería poder detectar casi todos los casos. ${ }^{(14)}$ Los estudios de laboratorio pueden ser normales o presentar leucocitos alterados en un $69,2 \%$, neutropenia $(6,0 \%)$, linfopenia $(3,0 \%)$ y neutrofilia $(4,6 \%)$. La proteína C reactiva (PCR) y la procalcitonina fueron altas sólo en el 13,6\% y el $10,6 \%$ de los casos, respectivamente. ${ }^{(15)}$

Se recomienda controlar el recuento de linfocitos y la PCR como signos de infección grave, mientras se utilizan los niveles de procalcitonina para detectar una posible coinfección bacteriana. Es común también encontrar elevación de las transaminasas hepáticas y es importante evaluar la presencia de otros marcadores de gravedad 
como el dímero $\mathrm{D}$, niveles de ferritina, de interleucina-6, presencia de trombocitopenia (esta última en adultos es un marcador de mal pronóstico). ${ }^{(15,16)}$

\section{Hallazgos radiológicos}

Los hallazgos de la radiografía de tórax en los niños parecen ser diferentes en comparación con los adultos. En EUA, Philip Zachariah y col realizaron radiografía de tórax a 36 niños positivos para Saros-Cov2, encontrando que el $69 \%$ presentaban opacidades bilaterales en vidrio despulido, 25\% derrame pleural, 22\% consolidación focal y neumotórax un 5\%. Hubo 5 pacientes sin síntomas respiratorios que presentaban infiltrados bilaterales. ${ }^{(17)}$

En los pacientes a los cuales se les ha realizado una tomografía axial computarizada de tórax (TAC) se ha observado la presencia de opacidades en vidrio despulido hasta en el 50\% de los pacientes, en un $18.7 \%$ existen imágenes de consolidación unilateral y en el $12.3 \%$ afección bilateral ${ }^{(7)}$. La presencia de una imagen de consolidación con el signo de halo circundante, se considera típica de los pacientes pediátricos ya que se ha encontrado hasta en el $50 \%$ de los pacientes, otros hallazgos menos frecuentes son nódulos y las imágenes en empedrado "crazy paving". ${ }^{(18,19)}$ El lóbulo superior derecho es el mas afectado en un $41 \%$, seguido por el lóbulo superior izquierdo (27\%); la afección multilobar es lo más frecuente en hasta $68 \%$ seguido por la focal $14 \%$ y la manifestación difusa en solo $9 \%$, estas últimas de predominio periférico. ${ }^{(16,19)}$ Con estos hallazgos podemos decir que la TAC de tórax es un auxiliar de diagnóstico útil para detectar cambios radiológicos asociados a COVID-19, pero no son signos específicos de SARS-CoV-2; el diagnóstico entonces siempre debe complementarse con las manifestaciones clínicas y resultados de laboratorio para hacer el diagnostico. ${ }^{(16)}$ Se ha utilizado el ultrasonido (USG) para ver cambios que nos puedan orientar al diagnostico de COVID-19. Las ventajas de utilizar el USG es que puede ser realizado en la cama del paciente, minimizar la necesidad de trasladarlo y disminuye el riesgo potencial de infección para el personal de salud. Históricamente se ha utilizado para definir anormalidades en la relación tejido/aire a nivel pulmonar, cuando la proporción entre aire, tejido, fluido $\mathrm{u}$ otros componentes biológicos se altera, aparecen las conocidas líneas B. ${ }^{(20)}$ Hay algunos estudios que han propuesto la utilización del USG en pacientes pediátricos con COVID-19; en un hospital de roma se realizó USG a 10 pacientes confirmados por PCR para SARS-CoV-2, y se encontraron; artefactos de verticales (70\%), irregularidades pleurales (60\%), áreas de pulmón blanco (10\%) y consolidaciones subpleurales (10\%). No se encontraron casos de derrames pleurales. En este estudio concluyen que puede ser una alternativa a la TAC ya que no requiere sedación del paciente. ${ }^{(21)}$

Las manifestaciones clínicas y hallazgos radiológicos de la COVID-19 pueden orientarnos al diagnóstico de infección por SARS-CoV-2, pero no son especificas de la enfermedad, ante la sospecha de otras infecciones concomitantes se debe valorar la presencia de coinfecciones, en un estudio de niños con COVID-19 se informó que de 74 pacientes a un $46 \%$ se les realizaron pruebas de detección para otros patógenos respiratorios obteniendo que el 51,4\% tenían alguna coinfección; la más frecuente fue infección por Micoplasma en un $84.2 \%$, seguido de virus sincitial respiratorio en un $15.8 \%$, y con el mismo porcentaje infección por el virus de EpsteinBarr, y citomegalovirus (CMV), y solo 5.3\% con influenza A y/o B. ${ }^{(22)}$ No existe un tratamiento específico contra SARS-CoV-2 hasta el momento. Hay muchos reportes en la literatura de los posibles medicamentos que podrían ser útiles que se escapan de la revisión de este artículo. Las estrategias terapéuticas para los pacientes pediátricos se basan en gran medida en las experiencias de los pacientes adultos. Se debería considerar un tratamiento estandarizado para los pacientes pediátricos en cada país y centro hospitalario mientras se realicen ensayos clínicos controlados para poder definir cuales son las mejore opciones de tratamiento para disminuir la mortalidad y secuelas de la enfermedad. En conclusión, las manifestaciones pulmonares asociadas al Sar-Cov2 en niños puede ir desde 
asintomáticos y leves que son las presentaciones mas frecuentes hasta una neumonía grave con falla multiorgánica que podrían ser fatal, en este momento cualquier síntoma respiratorio de vía aérea superior e inferior hay que descartar siempre una infección por COVID-19 (aunque en niños las manifestaciones son diferentes y pudieran debutar con manifestaciones cutáneas, gastrointestinales u otras) con pruebas moleculares o serológicas, acompañada de exámenes laboratoriales y radiológicos para poder evitar complicaciones en el paciente.

\section{REFERENCIAS BIBLIOGRÁFICAS}

1. GBD 2016 Lower Respiratory Infections Collaborators. Estimates of the global, regional, and national morbidity, mortality, and aetiologies of lower respiratory infections in 195 countries, 1990-2016: a systematic analysis for the Global Burden of Disease Study 2016. The Lancet Infectious Diseases. 2018 September 19; 18(11): 1191-1210.

2. Jin Y, Yang H, Ji W, Wu W, Chen S, Zhang W, et al. Virology, Epidemiology, Pathogenesis, and Control of COVID-19. Viruses. 2020 March 27; 12(4): 372 .

3. Song R, Preston G, Yosypiv IV. Ontogeny of Angiotensin-Converting Enzyme 2. Pediatric Research. 2012 January; 71(1): 13-19.

4. Liu Y, Yang Y, Zhang C, Huang F, Wang F, Yuan J, et al. Clinical and biochemical indexes from 2019-nCoV infected patients linked to viral loads and lung injury. Sci China Life Sciences. 2020 February 9; 63(3): 364-374.

5. Cavallo F, Rossi N, Chiarelli F. Novel Coronavirus Infection and Children. Acta Biomedica. 2020 May; 91(2): 172-176.

6. Wang D, Hu B, Hu C, Zhu F, Liu X, Zhang J, et al. Clinical Characteristics of 138 Hospitalized Patients With 2019 Novel Coronavirus-Infected Pneumonia in Wuhan, China. JAMA. 2020
February; 323(11): 1061-1069.

7. Vaduganathan $M$, Vardeny $O$, Michel $T$, McMurray JJV, Pfeffer MA, Solomon SD. ReninAngiotensin-Aldosterone System Inhibitors in Patients with Covid-19. English Journal of Medicine. 2020 April 23; 382: 1653-1659.

8. Chang TH, Wu JL, Chang LY. Clinical characteristics and diagnostic challenges of pediatric COVID-19: A systematic review and meta-analysis. Journal of the Formosan Medical Association. 2020 May; 119(5): 982-989.

9. Ludvigsson J. Systematic review of COVID-19 in children shows milder cases and a better prognosis than adults. Acta Pediatrica. 2020 March 23; 109(6): 1088-1095.

10. R.Kammoun , K.Masmoudi. Paediatric aspects of COVID-19: An update. Respiratory Medicine and Research. 2020.

11. Dong Y, Mo X, Hu Y, Qi X, Jiang F, Jiang Z, et al. Epidemiology of COVID-19 Among Children in China. Pediatrics. 2020 June; 145(6)

12. Dhochak N, Singhal T, Kabra SK, Lodha R. Pathophysiology of COVID-19: Why Children Fare Better than Adults? Indian Journal of Pediatrics. 2020 May;: 1-10.

13. Carsana L, Sonzogni A, Nasr A, Rossi R, Pellegrinelli A, Zerbi P, et al. Pulmonary postmortem findings in a large series of COVID-19 cases from Northern Italy. The Lancet Infectous Diseases. 2020 June.

14. Wang W, Xu Y, Gao R, Lu R, Han K, Wu G, et al. Detection of SARS-CoV-2 in Different Types of Clinical Specimens. JAMA. 2020 March; 323(18): 1843-1844.

15. Henry BM, Lippi G, Plebani M. Laboratory abnormalities in children with novel coronavirus disease 2019. Clinical chemistry and laboratory medicine. 2020 June; 58(7): 1135-1138. 
16. Balasubramanian S, Rao NM, Goenka A, Roderick M, Ramanan AV. Coronavirus Disease 2019 (COVID-19) in Children - What We Know So Far and What We Do Not. Indian Pediatrics. 2020 May 15; 57(5): 435-442.

17. Zachariah P, Johnson CL, Halabi KC, Ahn D, Sen AI, Fischer A, et al. Epidemiology, Clinical Features, and Disease Severity in Patients With Coronavirus Disease 2019 (COVID-19) in a Children's Hospital in New York City, New York. JAMA. 2020 June 3.

18. Xia W, Shao J, Guo Y, Peng X, Li Z, Hu D. Clinical and CT Features in Pediatric Patients With COVID-19 Infection: Different Points From Adults. Pediatric pulmonology. 2020 May; 55(5): 1169-1174.

19. Li B, Shen J, Li L, Yu C. Radiographic and Clinical Features of Children With Coronavirus Disease (COVID-19) Pneumonia. Indian Pediatrics. 2020 May; 57(5): 423-426.

20. Soldati $G$, Smargiassi $A$, Inchingolo $R$, Buonsenso D, Perrone T, Briganti DF, et al. Is There a Role for Lung Ultrasound During the COVID-19 Pandemic? Journal of ultrasound in medicine. 2020 July; 39(7): 1459-1462.

21. Musolino AM, Supino MC, Buonsenso D, Ferro V, Valentini P, Magistrelli A, et al. Lung Ultrasound in Children With COVID-19: Preliminary Findings. Ultrasound in medicine \& biology. 2020 May 3; S0301-5629(20).

22. Wu Q, Xing Y, Shi L, Li W, Gao Y, Pan S, et al. Coinfection and Other Clinical Characteristics of COVID-19 in Children. Pediatrics. 2020 June; 145(6).

23. Kunling S, Yonghong Y, Tianyou W, Dongchi Z, Yi J, Runming J, et al. Expert consensus on diagnosis, treatment and prevention of novel coronavirus infection in pediatrics (the first edition). Chinese Journal of Applied Clinical Pediatrics. 2020; 35(2): 81-85. 